\title{
Strong Normalization in a Non-Deterministic Typed Lambda-Calculus
}

\author{
Philippe de Groote \\ INRIA-Lorraine - CRIN - CNRS \\ Campus Scientifique - B.P. 239 \\ 54506 Vandœuvre-lès-Nancy Cedex - FRANCE \\ e-mail: degroote@loria.fr
}

\begin{abstract}
In a previous paper [4], we introduced a non-deterministic $\lambda$-calculus $(\lambda$-LK $)$ whose type system corresponds exactly to Gentzen's cut-free LK [9]. This calculus, however, cannot be provided with a computational interpretation. Some of the constructs act as oracles and, for this reason, it is not possible to define an effective notion of reduction. In the present paper, we address this problem. We consider a weak version of the implicative fragment of $\lambda$-LK, and we define for it a relation of reduction that models, at the level of the terms, the appropriate proof-theoretic notion of proof reduction. This reduction relation satisfies several properties of interest, among others, the property of strong normalization. We prove this last result by using a reducibility argument à la Tait.
\end{abstract}

\section{Introduction}

In recent works, several authors have addressed the problem of extending the formulae-astypes principle to classical logic, in order to express the computational content of classical proofs $[2,10,12,17,18,19]$. This problem cannot have a unique solution because one knows that the technical content of the formulae-as-types principle, namely the Curry-Howard isomorphism $[3,11,14,21]$, is strongly related to the constructive aspects of intuitionistic logic. Therefore, when dealing with classical logic, one has to drop some of the properties that exist in the intuitionistic case. Then, according to the properties that one drops, the solutions proposed to the problem are different. For instance, cut-elimination in classical logic is non-deterministic. Hence, in order to obtain a classical calculus that satisfies the Church-Rosser property, one has to restore confluence by defining some appropriate reduction strategy.

Yet as another proposal, we introduced, in a previous paper [4], a non-deterministic $\lambda$-calculus $(\lambda$-LK) whose type system corresponds to Gentzen's cut-free LK [9]. Our goal, 
however, was not to express the computational content of classical logic but rather to provide a model in which the non-deterministic and non-constructive aspects of classical logic can be explained.

Our idea was to introduce a dummy element $(\perp)$ to allow for the rule of right-weakening, a non-deterministic binary choice operators $(-\rrbracket-)$ to allow for the rule of right-contraction, and a non constructive choice operator akin to Hilbert's $\epsilon$ [16] to allow for the classical rule of right-implication. These features are highly non-deterministic. Moreover, because of the operator $\epsilon$ that acts as an oracle, this non-determinism must be interpreted in an angelic way. Because of this, it is not possible to provide $\lambda$-LK with usual reduction rules that would model faithfully the proof-theoretic process of cut-elimination.

In this paper, we study more deeply this last problem and we show that the angelic nature of $\lambda$-LK is only related to the operator $\epsilon$. In contrast, the non-determinism that arises from the right structural rules may be handled by using appropriate reduction rules.

Technically, we consider a weakening of the implicative fragment of $\lambda$-LK that is obtained by dropping the $\epsilon$ operator. The resulting system, that we have called $\lambda$-wLK $\rightarrow$ (read "weak lambda-LK"), remains a non-deterministic typed $\lambda$-calculus. Its typing system is a sequent calculus whose sequents may be manifold concluded. The underlying logic, however, is intuitionistic because of some proviso on the introduction rule of implication. It is then possible to define a reduction relation between the terms of $\lambda$-wLK that models a proof-theoretic notion of proof reduction. This reduction relation satisfies the properties of subject reduction, of Church-Rosser, and of strong normalization.

We prove this last property by using Gallier-Koletsos version of reducibility, as described in [8]. J. Gallier claims that this variant of the reducibility method is smoother than other versions in the sense that it can be easily adapted to various typed calculi. Our proof is also an illustration of this fact.

\section{Problems with $\lambda$-LK}

The idea behind the design of $\lambda$-LK [4] was to add three new constructs to the ones of the $\lambda$-calculus: a special constant $(\perp)$ and two non-deterministic choice operators $(-\square-$ and $\epsilon(-))$. The intuitive meaning of these constructs is the following:

$\perp$ is a fictitious proof; in some sense, it stands for something that does not exist;

- \ - is a binary choice operator whose non-determinism is angelic; the value of $M \square N$ is the value of $M$ or the value of $N$ but cannot be fictitious unless the values of both $M$ and $N$ are fictitious;

$\epsilon(-)$ is a choice operator akin to Hilbert's $\epsilon$ [16]; if $\alpha$ is a non-empty type, $\epsilon(\alpha)$ stands for some element of $\alpha$; if $\alpha$ is empty, $\epsilon(\alpha)$ is a fictitious term.

These constructs are used to decorate the formulas occurring in the right-hand side of a classical sequent. More precisely, they are used in the right rules as follows: 


$$
\begin{array}{cc}
\frac{\Gamma \vdash \Theta}{\Gamma \vdash \Theta, \perp: \alpha} & \text { (weakening - right) } \\
\frac{\Gamma \vdash \Theta, M: \alpha, N: \alpha}{\Gamma \vdash \Theta, M \llbracket N: \alpha} & \text { (contraction - right) } \\
\frac{x: \alpha, \Gamma \vdash \Theta, M: \beta}{\Gamma \vdash \Theta[x:=\epsilon(\alpha)], \lambda x: \alpha \cdot M: \alpha \rightarrow \beta} & \text { (implication - right) }
\end{array}
$$

As far as cut-free proofs are concerned, the system $\lambda$-LK is satisfactory. If one allows for cuts, however, there is a mismatch between $\beta$-reduction, which acts at the local level of the terms, and cut elimination, which acts at the global level of the sequents. This can be shown by considering the main step of cut elimination:

$$
\begin{aligned}
& \begin{array}{ccc}
\vdots & \vdots & \vdots \\
\Pi_{1} & \Pi_{2} & \Pi_{3}
\end{array}
\end{aligned}
$$

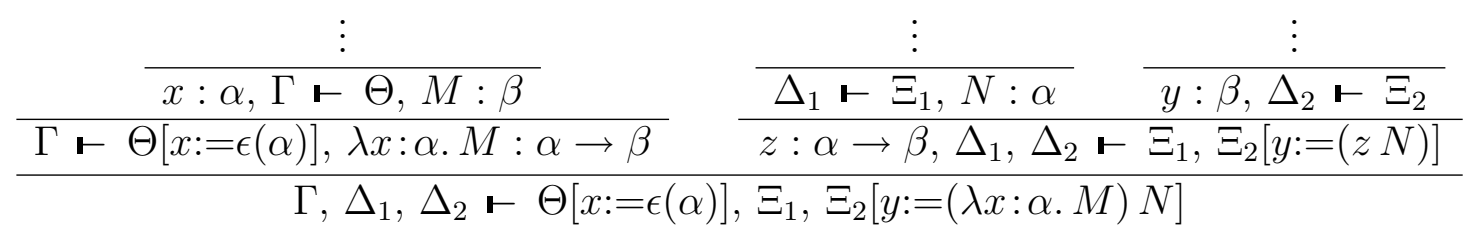

reduces to:

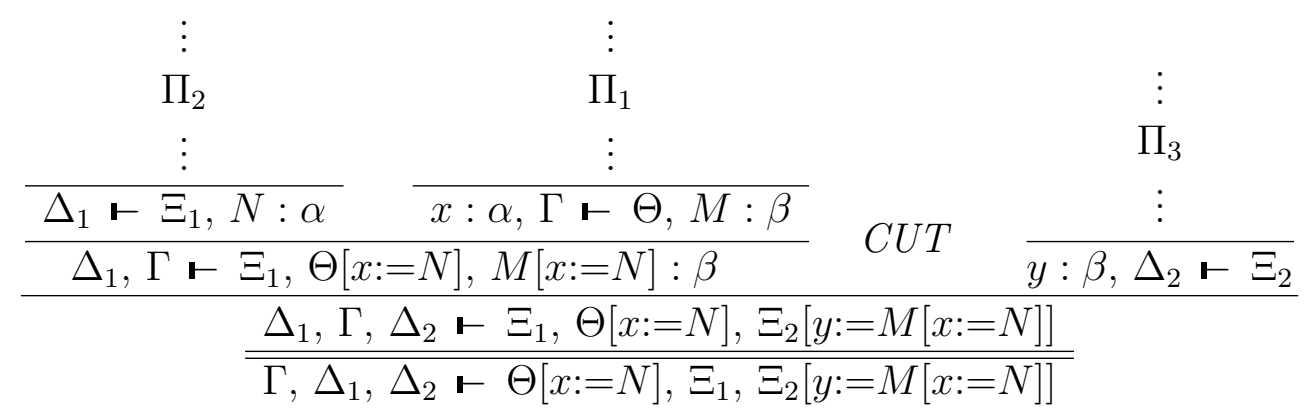

The sequent obtain by cut elimination is the following one:

$$
\Gamma, \Delta_{1}, \Delta_{2} \vdash \Theta[x:=N], \Xi_{1}, \Xi_{2}[y:=M[x:=N]]
$$

On the other hand, the process of $\beta$-reduction yields the following result:

$$
\Gamma, \Delta_{1}, \Delta_{2} \vdash \Theta[x:=\epsilon(\alpha)], \Xi_{1}, \Xi_{2}[y:=M[x:=N]]
$$

In fact the problem of providing $\lambda$-LK with an appropriate reduction theory is twofold. On the one hand, as suggested by the above example, we should allow for non-effective reductions related to the $\epsilon$-construct. On the other hand, we must also provide reduction rules related to the constant $\perp$ and the operator $(-\rrbracket-)$.

In this paper, we address the second question by considering the system $\lambda$-LK without the operator $\epsilon$. 


\section{Formal Definition of $\lambda$-wLK $\rightarrow$}

In this section, we introduce formally $\lambda$-wLK $\rightarrow$, which is obtained from the implicative fragment of $\lambda$-LK by dropping the $\epsilon$ operator. For more motivations about the design of $\lambda$-LK we report the reader to our previous work [4].

Definition 3.1 (Syntax of Raw Terms) Let $\mathcal{A}$ be a countably infinite set of type-variables. The set $\mathcal{T}$ of types of $\lambda$-wLK $\rightarrow$ is inductively defined as follows:

(i) if $a \in \mathcal{A}$ then $a \in \mathcal{T}$;

(ii) if $\alpha, \beta \in \mathcal{T}$ then $(\alpha \rightarrow \beta) \in \mathcal{T}$.

Let $\mathcal{X}$ be a countably infinite set of term-variables. The set $\Lambda_{\mathrm{wLK}}$ of terms of $\lambda$-wLK $\rightarrow$ is inductively defined as follows:

(i) $\perp_{\alpha} \in \Lambda_{\mathrm{wLK}} \quad$ for each $\alpha \in \mathcal{T}$;

(ii) if $x \in \mathcal{X}$ then $x \in \Lambda_{\mathrm{wLK}}$;

(iii) if $x \in \mathcal{X}, \alpha \in \mathcal{T}$ and $M \in \Lambda_{\mathrm{wLK}}$ then $(\lambda x: \alpha . M) \in \Lambda_{\mathrm{wLK}}$.

(iv) if $M, N \in \Lambda_{\mathrm{wLK}}$ then $(M N) \in \Lambda_{\mathrm{wLK}}$;

(v) if $M, N \in \Lambda_{\mathrm{wLK}}$ then $(M \rrbracket N) \in \Lambda_{\mathrm{wLK}}$.

The relation of syntactic identity $(\equiv)$ between the terms of $\lambda$-wLK $\rightarrow$ is defined as the finest congruence containing the relation of $\alpha$-conversion and satisfying the three following axioms:

$$
((M \rrbracket N) \rrbracket O) \equiv(M \rrbracket(N \rrbracket O)) \quad(M \rrbracket N) \equiv(N \rrbracket M) \quad(M \rrbracket M) \equiv M
$$

From now on, any term $M$ will stand for its class of equivalence modulo $\equiv$. The notation

$$
\prod_{i \in n} M_{i}
$$

will stand for the term $\left(M_{0} \rrbracket M_{1} \rrbracket \cdots \rrbracket M_{n-1}\right)$, where no $M_{i}$ is of the form $M_{i}^{\prime} \rrbracket M_{i}^{\prime \prime}$. Nevertheless, in order to simplify the notation, we will sometimes write $M_{1} \rrbracket M_{2}$ to mean $\rrbracket_{i \in n} M_{i}$.

We use $\alpha, \beta, \gamma, \ldots$ to denote types and $M, N, O, \ldots$ to denote terms. Expressions of the form $M: \alpha$ are called statements, or declarations when $M$ is a variable. A sequence of declarations $\left(x_{1}: \alpha_{1}, \ldots, x_{n}: \alpha_{n}\right)$ where all the $x_{i}$ 's are different is called a context. We use $\Gamma, \Delta, \ldots$ to denote contexts, and $\Theta, \Xi, \ldots$ to denote sequences of statements. The expression $M[x:=N]$ denotes the result of substituting $N$ for the free occurrences of $x$ in $M$. If $\Theta$ is the sequence $\left(M_{1}: \alpha_{1}, \ldots, M_{n}: \alpha_{n}\right)$ then $\Theta[x:=N]$ is the sequence $\left(M_{1}[x:=N]\right.$ : $\left.\alpha_{1}, \ldots, M_{n}[x:=N]: \alpha_{n}\right)$. 
Definition 3.2 (Type Assignment) The type assignment system of $\lambda$-wLK $\rightarrow$ consists of the following rules:

$$
\text { (i) } \Gamma \vdash \Gamma
$$

$$
\begin{aligned}
& \text { (ii) } \frac{\Gamma, x: \alpha, y: \beta, \Delta \vdash \Theta}{\Gamma, y: \beta, x: \alpha, \Delta \vdash \Theta} \\
& \text { (iii) } \frac{\Gamma \vdash \Theta, M: \alpha, N: \beta, \Xi}{\Gamma \vdash \Theta, N: \beta, M: \alpha, \Xi} \\
& \text { (iv) } \frac{\Gamma \vdash \Theta}{x: \alpha, \Gamma \vdash \Theta} \\
& \text { (v) } \frac{\Gamma \vdash \Theta}{\Gamma \vdash \Theta, \perp_{\alpha}: \alpha} \\
& \text { (vi) } \frac{y: \alpha, z: \alpha, \Gamma \vdash \Theta}{x: \alpha, \Gamma \vdash \Theta[y:=x][z:=x]} \\
& \text { (vii) } \frac{\Gamma \vdash \Theta, M: \alpha, N: \alpha}{\Gamma \vdash \Theta, M \rrbracket N: \alpha} \\
& \text { (viii) } \frac{\Gamma \vdash \Theta, M: \alpha \rightarrow \beta \Delta \vdash \Xi, N: \alpha}{\Gamma, \Delta \vdash \Theta, \Xi, M N: \beta} \\
& \text { (ix) } \frac{x: \alpha, \Gamma \vdash \Theta, M: \beta}{\Gamma \vdash \Theta, \lambda x: \alpha \cdot M: \alpha \rightarrow \beta} \text { if } x \text { does not occur free in } \Theta \text {. }
\end{aligned}
$$

In Rule (iv) and Rule (vi), the variable $x$ must be fresh. Let $M \in \Lambda_{\mathrm{wLK}}$. We say that $M$ is a well-typed term of $\lambda$-wLK $\rightarrow$ if and only if there exist a context $\Gamma$, a sequence of statements $\Theta$, and a type $\alpha$ such that the sequent $\Gamma \vdash \Theta, M: \alpha$ is derivable.

The non-occurrence condition of Rule (ix) is sufficient to force our system to remain intuitionistic. This is not surprising because there is a strong connection between semantic tableaux and our system. For classical logic, the method of semantic tableaux corresponds exactly to the sequent system G1 of Kleene [15]. For intuitionistic logic there is no similar correspondence: in intuitionistic tableaux one deals with sets of $F$-signed formulas [5], while the succedent of any intuitionistic sequent contains at most one formula. The only difference between intuitionistic and classical tableaux for propositional logic lies in the treatment of an $F$-signed implication or negation. The non-occurrence condition of Rule (ix) can be seen as a refinement of a similar proviso that exists in the case of intuitionistic tableaux.

Proposition 3.3 Let the sequent $\left(x_{i}: \alpha_{i}\right)_{i \in n} \vdash\left(M_{j}: \beta_{j}\right)_{j \in m}$ be derivable according to the system of Definition 3.2. Then the formula

$$
\bigwedge_{i \in n} \alpha_{i} \rightarrow \bigvee_{j \in m} \beta_{j}
$$

is intuitionistically valid. 


\section{Term and Proof Reduction}

Prawitz has introduced, in the framework of Gentzen's NJ [9], the notion of normal proof [20]. Given any proof in NJ, its normal form may be reached by a process of proof reduction, which corresponds, through the isomorphism of Curry-Howard, to a relation of reduction between $\lambda$-terms.

In NJ, a proof is not in normal form when the principal formula of an elimination rule is obtained as the conclusion of the corresponding introduction rule. In the case of implication, such a proof corresponds to a $\beta$-redex. In our system, a formula may also be introduced by weakening and this possibility gives rise to additional reduction steps:

$$
\frac{\Gamma \vdash \Theta}{\frac{\Gamma \vdash \Theta, \perp_{(\alpha \rightarrow \beta)}: \alpha \rightarrow \beta}{\Gamma, \Delta \vdash \Theta, \Xi, \perp_{(\alpha \rightarrow \beta)} M: \beta}} \quad \begin{gathered}
\vdots \\
\frac{\Delta}{\Gamma, \Xi, M: \alpha}
\end{gathered} \rightarrow \frac{\Gamma, \Delta \vdash \Theta, \Xi}{\Gamma, \Delta \vdash \Theta, \Xi, \perp_{\beta}: \beta}
$$

We must also allow for structural reductions that are related to the commutation of the right-contraction with application. Putting these ideas together, we are led to the following definition.

Definition 4.1 (Reduction) The relation of one-step reduction $(\rightarrow)$ is the smallest relation containing the following redex-contractum pairs and compatible with the term formation rules.

(i) $\quad(\lambda x: \alpha . M) N \rightarrow M[x:=N]$

(ii) $\perp_{(\alpha \rightarrow \beta)} M \rightarrow \perp_{\beta}$

(iii) $\perp_{\alpha} \rrbracket M \rightarrow M$

(iv) $\left(\rrbracket_{i \in n} M_{i}\right) N \rightarrow \rrbracket_{i \in n} M_{i} N$

The relation of strict reduction $(\stackrel{+}{\rightarrow})$ is the transitive closure of the relation of one-step reduction. Finally, the relation of reduction $(\rightarrow)$ is the reflexive closure of the relation of one-step reduction.

The reduction relation is such that the reducts of a well-typed term are typable with respect to the same context and within the same sequence of statements. This result is a piece of evidence that the reduction relation between terms models faithfully the process of proof reduction.

Proposition 4.2 (Subject Reduction) Let $\Gamma \vdash \Theta, M: \alpha$ be derivable and let $M \rightarrow M^{\prime}$. Then the sequent $\Gamma \vdash \Theta, M^{\prime}: \alpha$ is derivable.

The relation of reduction also satisfies the Church-Rosser property. 


\section{Strong Normalization}

In this section we establish the main result of this paper, i.e., the strong normalization of the well-typed terms of $\lambda$-wLK $\rightarrow$. To this end we introduce a weaker notion of well-typedness. For technical reasons, this definition is a definition à la Church, and we assume the existence of a family $\left(\mathcal{X}_{\alpha}\right)_{\alpha \in \mathcal{T}}$, where each $\mathcal{X}_{\alpha}$ is a countably infinite set of variables of type $\alpha$.

Definition 5.1 The family of sets $\left(\Lambda_{\alpha}\right)_{\alpha \in \mathcal{T}}$ of terms of type $\alpha$ is inductively defined as follows:

(i) $\perp_{\alpha} \in \Lambda_{\alpha}$,

(ii) if $x \in \mathcal{X}_{\alpha}$ then $x \in \Lambda_{\alpha}$,

(iii) if $x \in \mathcal{X}_{\alpha}$ and $M \in \Lambda_{\beta}$ then $\lambda x: \alpha . M \in \Lambda_{(\alpha \rightarrow \beta)}$,

(iv) if $M \in \Lambda_{(\alpha \rightarrow \beta)}$ and $N \in \Lambda_{\alpha}$ then $(M N) \in \Lambda_{\beta}$.

(v) if $M, N \in \Lambda_{\alpha}$ then $(M \rrbracket N) \in \Lambda_{\alpha}$.

Let $\Gamma$ be an environment that agrees with $\left(\mathcal{X}_{\alpha}\right)_{\alpha \in \mathcal{T}}$, i.e., an environment such that $x \in \mathcal{X}_{\alpha}$ whenever $x: \alpha$ occurs in $\Gamma$. It is easy to show that for every terms $M_{i}$ and every types $\alpha_{i}(0 \leq i<n)$ such that

$$
\Gamma \vdash\left(M_{i}: \alpha_{i}\right)_{i \in n}
$$

one has that $M_{i} \in \Lambda_{\alpha_{i}}{ }^{1}$. Therefore to show that every term well-typed according to Definition 3.2 is strongly normalizable with respect to the reduction relation of definition 4.1 , we may show that every term in the family $\left(\Lambda_{\alpha}\right)_{\alpha \in \mathcal{T}}$ satisfies the same normalization property.

To show that every term in the family $\left(\Lambda_{\alpha}\right)_{\alpha \in \mathcal{T}}$ is strongly normalizable (SN, for short), we use a reducibility argument $[7,8,11]$.

Definition 5.2 (Reducibility) The sets $\llbracket \alpha \rrbracket$ of reducible terms of type $\alpha$ are inductively defined as follows:

(i) $\llbracket \alpha \rrbracket=\left\{M \in \Lambda_{\alpha} \mid M\right.$ is $\left.S N\right\} \quad$ when $\alpha$ is atomic,

(ii) $\left[\left[\alpha \rightarrow \beta \rrbracket=\left\{M \in \Lambda_{(\alpha \rightarrow \beta)} \mid\right.\right.\right.$ for all $N \in \llbracket[\alpha \rrbracket,(M N) \in \llbracket \beta \rrbracket\}$.

Our strong normalization proof is adapted from [8]. Following Gallier, we define the notions of I-term, simple term, and stubborn term.

Definition 5.3 An I-term is a term of the form $\perp_{\alpha}, \lambda x: \alpha . M$, or $(M \llbracket N)$. A simple terms is a term that is not an I-term (that is either a variable or an application). A stubborn term is a term that reduces (in zero or more steps) only to simple terms.

\footnotetext{
${ }^{1}$ The converse, however, does not hold
} 
In fact the I-terms are the terms that correspond to introduction rules. Their key property is that they give rise to a redex when applied to another term. Therefore to show that an application $M Q$ is $\mathrm{SN}$, it is sufficient to show that, for any I-term $N$ such that $M \rightarrow N, N Q$ is $\mathrm{SN}$. This is an instance of the property (P3) in [8].

The stubborn terms, on the other hand, when applied to another term cannot give rise to a redex, even after reduction. Therefore, when a stubborn term is applied to another term, the resulting application and all its possible reducts are stubborn.

The strong normalization proofs consists of five lemmas. The first one concerns the following closure properties of the sets of reducible terms:

(R1) if $M \in \llbracket \alpha]$, then $M$ is $\mathrm{SN}$;

(R2) if $M \in \llbracket \alpha \rrbracket$ and $M \rightarrow N$ then $N \in \llbracket \alpha \rrbracket$.

(R3) if $M$ is simple, and if $N \in \llbracket \alpha \rrbracket$ for any I-term $N$ such that $M \rightarrow N$, then $M \in \llbracket \alpha \rrbracket$.

Remark that the second condition of Property (R3) is vacuously satisfied when $M$ is stubborn. Therefore, Property (R3) implies that every stubborn term is reducible.

Lemma 5.4 For every type $\alpha$, the set of reducible terms $\llbracket \alpha \rrbracket$ satisfies Properties (R1), (R2), and (R3).

Proof. The proof is by induction on the structure of $\alpha$.

Base: $\alpha$ is an atomic type. The three properties follow easily.

Induction step: $\alpha \equiv(\beta \rightarrow \gamma)$.

(R1) Let $x \in \mathcal{X}_{\beta}$. By induction hypothesis (R3), $x \in \llbracket \beta \rrbracket$. Hence, by definition of reducibility, $M x \in \llbracket \gamma \rrbracket$. Therefore, by induction hypothesis (R1), $M x$ is SN and so is $M$.

(R2) For any $O \in[\beta]]$ one has that $M O \in[[\gamma]$ and that $M O \rightarrow N O$. Therefore, by induction hypothesis (R2), for any $O \in[[\beta]$ one has that $N O \in \llbracket \gamma \rrbracket$. Hence, by definition of reducibility, $N \in \llbracket \beta \rightarrow \gamma \rrbracket$.

(R3) Let $Q \in \llbracket \beta \rrbracket$. We must show that $M Q$ is reducible.

If $M$ is stubborn, so is $M Q$. Therefore, by induction hypothesis (R3), $M Q \in \llbracket \gamma \rrbracket$.

Assume that $M$ is not stubborn. We proceed by induction on $\gamma$.

If $\gamma$ is atomic, we must show that $M Q$ is $\mathrm{SN}$. By assumption, $N \in \llbracket \beta \rightarrow \gamma \rrbracket$ for any I-term $N$ such that $M \rightarrow N$. Hence $N Q \in \llbracket \gamma \rrbracket$. Therefore $N Q$ is SN by induction hypothesis (R1), and so is $M Q$. 
If $\gamma$ is not atomic, we show that $M Q \in[\gamma]$, by using induction hypothesis (R3). On the one hand we have that $M Q$ is simple. On the other hand, if there is any I-term $P$ to which $M Q$ reduces, the reduction is necessarily of one the following forms:

$$
\begin{aligned}
& M Q \stackrel{+}{\rightarrow} \perp_{\beta \rightarrow \gamma} Q_{1} \rightarrow \perp_{\gamma} \equiv P \\
& M Q \stackrel{+}{\rightarrow}\left(\lambda x: \beta . M_{1}\right) Q_{1} \rightarrow M_{1}\left[x:=Q_{1}\right] \rightarrow P \\
& M Q \stackrel{+}{\rightarrow}\left(M_{1} \rrbracket M_{2}\right) Q_{1} \rightarrow M_{1} Q_{1} \rrbracket M_{2} Q_{1} \rightarrow P \\
& M Q \stackrel{+}{\rightarrow}\left(\perp_{\alpha} \rrbracket M_{2}\right) Q_{1} \rightarrow M_{2} Q_{1} \rightarrow P
\end{aligned}
$$

where $M$ respectively reduces to the I-terms $\perp_{\beta \rightarrow \gamma}, \lambda x: \beta . M_{1}, M_{1} \rrbracket M_{2}$, or $\perp_{\alpha} \rrbracket M_{2}$; and $Q$ reduces to $Q_{1}$. Let $N$ be respectively $\perp_{\beta \rightarrow \gamma}, \lambda x: \beta . M_{1}, M_{1} \rrbracket M_{2}$, or $\perp_{\alpha} \rrbracket M_{2}$. By assumption $N \in \llbracket \beta \rightarrow \gamma \rrbracket$. On the other hand, by induction hypothesis (R2), $Q_{1} \in \llbracket \beta \rrbracket$. Therefore $N Q_{1} \in \llbracket[\gamma \rrbracket$. Hence, again by induction hypothesis (R2), $P \in \llbracket[\gamma \rrbracket$. This allows us to apply induction hypothesis (R3) and conclude that $M Q \in \llbracket \gamma \rrbracket$.

We have established that every reducible term is strongly normalizable. The next step, in a reducibility proof, is to establish that every term is reducible. To establish this second property, in the case of pure $\lambda$-terms, one needs first to prove a lemma about $\lambda$-abstraction. More generally, what is needed are such lemmas concerning each form of I-term. Thus we need here three lemmas.

Lemma 5.5 For every type $\alpha, \perp_{\alpha} \in \llbracket \alpha \rrbracket$.

Proof. The proof is by induction on $\alpha$.

If $\alpha$ is atomic, we have that $\perp_{\alpha}$ is SN.

If $\alpha$ is not atomic, the property follows by induction hypothesis and Property (R3).

Lemma 5.6 Let $\alpha$ be a type. If $M, N \in \llbracket \alpha \rrbracket$ then $(M \rrbracket N) \in \llbracket \alpha \rrbracket$.

Proof. The proof is by induction on $\alpha$.

At base type, we have that $M$ and $N$ are SN by Property (R1). Therefore, so is $(M \rrbracket N)$. Let $\alpha \equiv \beta \rightarrow \gamma$, and assume $Q \in \llbracket \beta \rrbracket$. If $(M \rrbracket N) Q$, which is simple, reduces to some I-term $P$, the reduction is necessarily of the following form:

$$
\begin{aligned}
& (M \rrbracket N) Q \rightarrow\left(M_{1} \rrbracket N_{1}\right) Q_{1} \rightarrow M_{1} Q_{1} \rrbracket N_{1} Q_{1} \rightarrow P \\
& (M \rrbracket N) Q \rightarrow\left(\perp_{\alpha} \rrbracket N_{1}\right) Q_{1} \rightarrow N_{1} Q_{1} \rightarrow P
\end{aligned}
$$

where $M \rightarrow M_{1}, N \rightarrow N_{1}$, and $Q \rightarrow Q_{1}$. By hypothesis, assumption, and Property (R2), $M_{1} \in \llbracket \beta \rightarrow \gamma \rrbracket, N_{1} \in \llbracket \beta \rightarrow \gamma \rrbracket$, and $Q_{1} \in \llbracket \beta \rrbracket$. Hence $M_{1} Q_{1}, N_{1} Q_{1} \in \llbracket \gamma \rrbracket$, and, by induction hypothesis, $\left(M_{1} Q_{1} \rrbracket N_{1} Q_{1}\right) \in \llbracket \gamma \rrbracket$. Therefore, by Property (R2), $P \in \llbracket \gamma \rrbracket$. This allows us to conclude by applying Property (R3).

Lemma 5.7 Let $x \in \mathcal{X}_{\alpha}$ and $M \in \Lambda_{\beta}$. If for all $N \in \llbracket \alpha \rrbracket$ one has that $M[x:=N] \in \llbracket \beta \rrbracket$, then $\lambda x: \alpha . M \in \llbracket \alpha \rightarrow \beta \rrbracket$. 
Proof. We must show that for every $N \in \llbracket \alpha \rrbracket$, one has that $(\lambda x: \alpha . M) N \in \llbracket \beta \rrbracket$. The proof is by induction on $\beta$.

Let $\beta$ be atomic. By hypothesis, $M[x:=N] \in \llbracket \beta \rrbracket$. Therefore, by Property $(\mathrm{R} 1), M[x:=N]$ is SN. It is easy to show that this implies that $(\lambda x: \alpha . M) N$ is SN (for instance, by using Substitution Lemma 3.2.4 in [1]).

Let $\beta$ be non-atomic. On the one hand, the term $(\lambda x: \alpha . M) N$ is simple. On the other hand, if $(\lambda x: \alpha . M) N$ reduces to some I-term $P$, the reduction must be of the following form:

$$
(\lambda x: \alpha \cdot M) N \rightarrow\left(\lambda x: \alpha \cdot M_{1}\right) N_{1} \rightarrow M_{1}\left[x:=N_{1}\right] \rightarrow P
$$

where $M \rightarrow M_{1}$ and $N \rightarrow N_{1}$. This implies that

$$
M[x:=N] \rightarrow M_{1}\left[x:=N_{1}\right]
$$

Hence, since $M[x:=N] \in \llbracket \beta \rrbracket$ by hypothesis, we have that $P \in \llbracket \beta \rrbracket$ by (R2). Therefore, by (R3), $\lambda x: \alpha . M \in[[\alpha \rightarrow \beta]$.

Lemma 5.8 Let $x_{i} \in \mathcal{X}_{\alpha_{i}}(1 \leq i \leq n)$ and let $M \in \Lambda_{\beta}$ be a term whose free variables are among the $x_{i}$ 's. If $N_{i} \in \llbracket\left[\alpha_{i}\right](1 \leq i \leq n)$, then $M\left[x_{1}:=N_{1}\right] \cdots\left[x_{n}:=N_{n}\right] \in \llbracket \beta \rrbracket$.

Proof. The proof is by induction on the structure of $M$. We write $M^{*}$ for $M\left[x_{1}:=N_{1}\right] \cdots\left[x_{n}:=N_{n}\right]$.

(i) $M \equiv \perp_{\beta}$.

By Lemma 5.5, $\perp_{\beta} \in \llbracket[\beta]$.

(ii) $M \equiv x_{i}$ and $\beta \equiv \alpha_{i}$.

$M^{*} \equiv N_{i}$ and, by hypothesis, $N_{i} \in \llbracket \alpha_{i} \rrbracket$.

(iii) $M \equiv \lambda x: \gamma . O$ and $\beta \equiv(\gamma \rightarrow \delta)$.

By induction hypothesis, for any $P \in[\gamma]]$, we have that $0^{*}[x:=P] \in[\delta \delta]$. Therefore, by Lemma 5.7, $\lambda x: \gamma . O^{*} \in \llbracket \beta \rrbracket$.

(iv) $M \equiv(O P)$ with $O \in \Lambda_{(\gamma \rightarrow \beta)}$ and $P \in \Lambda_{\gamma}$.

By induction hypothesis, $O^{*} \in \llbracket \gamma \rightarrow \beta \rrbracket$ and $P^{*} \in \llbracket \gamma \rrbracket$. Therefore, by definition of reducibility, $\left.\left(O^{*} P^{*}\right) \in \llbracket \beta\right]$.

(v) $M \equiv(O \rrbracket P)$.

By induction hypothesis, $O^{*}, P^{*} \in \llbracket \beta \rrbracket$. Therefore, by Lemma 5.6, $\left(O^{*} \rrbracket P^{*}\right) \in \llbracket \beta \rrbracket$.

Proposition 5.9 (Strong Normalization) All the well-typed terms of $\lambda$-wLK $\rightarrow$ are strongly normalizable.

Proof. By Lemma 5.8, taking $N_{i} \equiv x_{i}$, every well-typed term is reducible. Then, by Lemma 5.4, Property (R1), every well-typed term is SN. 


\section{$6 \quad$ Other Reduction Rules}

Reduction Rules (ii), (iii), and (iv) of Definition 4.1 model the way in which the right structural rules interact with the other rules. In Gentzen's LK, other interactions exist. For instance, right-contraction commutes with implication-introduction:

$$
\frac{\vdots}{\frac{\Gamma, \alpha \vdash \beta, \beta, \Theta}{\Gamma, \alpha \vdash \beta, \Theta}} \frac{\frac{\Gamma, \alpha \vdash \beta, \beta, \Theta}{\Gamma, \alpha \vdash \beta, \alpha \rightarrow \beta, \Theta}}{\frac{\Gamma \vdash \alpha \rightarrow \beta, \alpha \rightarrow \beta, \Theta}{\Gamma \vdash \alpha \rightarrow \beta, \Theta}}
$$

Therefore, one may think of the following additional reduction rules:

(v) $M \perp_{\alpha} \rightarrow \perp_{\beta}$

(in a context where $M$ is of type $\alpha \rightarrow \beta$ )

(vi) $\lambda x: \alpha . \perp_{\beta} \rightarrow \perp_{(\alpha \rightarrow \beta)}$

(vii) $M\left(\rrbracket_{i \in n} N_{i}\right) \rightarrow \rrbracket_{i \in n} M N_{i}$

(viii) $\lambda x: \alpha \cdot\left(\rrbracket_{i \in n} M_{i}\right) \rightarrow \rrbracket_{i \in n} \lambda x: \alpha \cdot M_{i}$

Strong Normalization still holds with these additional reduction rules (the proof of course is longer because there are numerous subcases, and the notion of I-term is more intricate). Besides, with the additional rules, the normal form of a term is either $\perp_{\alpha}$, or of the form $\rrbracket_{i \in n} M_{i}$ where each $M_{i}$ is a well-typed term of Church's simply typed $\lambda$-calculus. Moreover, when a sequent is derivable, there is a least one term whose normal form is not $\perp_{\alpha}$. This, together with subject reduction, proves Proposition 3.3, and gives us an effective procedure to extract natural deduction proofs from a term in our system.

There are, however, good reasons why we did not allow for the additional reduction rules. On the one hand, they correspond to a sort of awkward call-by-value strategy. Rule (v), for instance, amounts to a kind of strictness while Rule (vi) says that the functions must be completely evaluated. On the other hand, they distroy the confluence of the calculus.

\section{Conclusions and Future Work}

The results that are presented in this paper can be extended in several ways.

While we have worked in the implicative fragment, the other propositional connectives can be taken into account as we exposed in [4]. Then the extension of Definition 4.1 to the system containing all the connectives is rather systematic, and the proofs of the different lemmas and theorems may be adapted.

As we already mentioned, there is a strong connection with intuitionistic semantic tableaux [5]. By adapting Definition 3.2 we could define a typing system in which the 
derivation of a typing judgment can be interpreted as an intuitionistic tableau. Then, strongnormalization would give us an effective procedure to extract natural deduction proofs from semantic tableaux.

Our final goal remains to analyze classical proofs. Therefore we must waive the side condition that comes with the introduction rule for implication.

A first solution would be to reintroduce the $\epsilon$-operator. With respect to this, the results that we have obtained in this paper are encouraging. In particular, the strong normalization proof is not affected by the addition of the $\epsilon$-operator, which corresponds simply to the addition of new constants. This observation, however, makes only one half of the job because we must also provide reduction rules to deal with $\epsilon$. As we have stressed in the introduction, the natures of the non-determinisms related respectively to $\epsilon$ and to the right structural rules are different. In this paper, we have explained how to deal with the latter. In order to handle the former, we must accept to deal with reduction relations that are not confluent and not effective. For instance, we must accept reduction rules such as the following:

$$
\frac{\Gamma \vdash \Theta, \epsilon(\alpha): \alpha \quad \Gamma \vdash \Theta, M: \alpha}{\Gamma \vdash \Theta, \epsilon(\alpha) \rightarrow M: \alpha}
$$

A second solution consists in providing the $\lambda$-terms with some communication mechanism. Consider once more the introduction rule for implication:

$$
\frac{x: \alpha, \Gamma \vdash \Theta, M: \beta}{\Gamma \vdash \Theta, \lambda x: \alpha \cdot M: \alpha \rightarrow \beta}
$$

The problem, in the classical case, is that $x$ may occur free in $\Theta$. If one allows for oneproducer/many-consumers communication, the classical rule may become the following:

$$
\frac{x: \alpha, \Gamma \vdash \Theta,\left(N_{i}: \gamma_{i}\right)_{i \in n}, M: \beta}{\Gamma \vdash \Theta,\left([a ? x] \cdot N_{i}: \gamma_{i}\right)_{i \in n}, \lambda x: \alpha \cdot[a ! x] . M: \alpha \rightarrow \beta} \quad x \text { does not occur free in } \Theta .
$$

where $[a ? x]$ means input $x$ from channel $a$, and $[a ! x]$ means output $x$ on channel $a$. With such a rule, the sequent corresponding to a main cut reduces as follows:

$$
\begin{aligned}
& \Gamma \vdash \Theta,\left([a ? x] \cdot N_{i}: \gamma_{i}\right)_{i \in n}, \Xi[y:=(\lambda x: \alpha \cdot[a ! x] . M) N] \rightarrow \\
& \Gamma \vdash \Theta,\left([a ? x] \cdot N_{i}: \gamma_{i}\right)_{i \in n}, \Xi[y:=[a ! N] \cdot M[x:=N]] \rightarrow \\
& \Gamma \vdash \Theta,\left(N_{i}[x:=N]: \gamma_{i}\right)_{i \in n}, \Xi[y:=M[x:=N]]
\end{aligned}
$$

We are currently working on this solution.

\section{References}

[1] H.P. Barendregt. The lambda calculus, its syntax and semantics. North-Holland, revised edition, 1984. 
[2] R. Constable and C. Murthy. Finding computational content in classical proofs. In G. Huet and G. Plotkin, editors, Logical Frameworks, pages 341-362. Cambridge University Press, 1991.

[3] H.B. Curry and R. Feys. Combinatory Logic, Vol. I. North-Holland, 1958.

[4] Ph. de Groote. Denotations for Classical Proofs - Preliminary Results. In A. Nerode and M. Taitslin, editors, Second International Symposium on Logical Foundations of Computer Science, Tver'92, volume 620 of Lecture Notes in Computer Science, pages 105-116. Springer Verlag, 1992.

[5] M.C. Fitting. Intuitionistic Logic Model Theory and Forcing. North Holland Publishing Company, 1969.

[6] J.H. Gallier. Logic for Computer Science. John Wiley \& Sons, 1988.

[7] J.H. Gallier. On Girard's "Candidats de Réductibilité". In P. Odifreddi, editor, Logic and Computer Science, pages 123-203. Academic Press, 1990.

[8] J.H. Gallier. On the correspondence between proofs and $\lambda$-terms. In Ph. de Groote, editor, Cahiers du Centre de Logique (Université Catholique de Louvain), Volume 8, pages 55-138. Academia, Louvain-la-Neuve, 1995.

[9] G. Gentzen. Recherches sur la déduction logique (Untersuchungen über das logische schliessen). Presses Universitaires de France, 1955. Traduction et commentaire par R. Feys et J. Ladrière.

[10] J.-Y. Girard. A new constructive logic: Classical logic. Mathematical Structures in Computer Science, 1:255-296, 1991.

[11] J.-Y. Girard, Y. Lafont, and P. Taylor. Proofs and Types, volume 7 of Cambridge Tracts in Theoretical Computer Science. Cambridge University Press, 1989.

[12] T. G. Griffin. A formulae-as-types notion of control. In Conference record of the seventeenth annual ACM symposium on Principles of Programming Languages, pages 47-58, 1990.

[13] J.R. Hindley and J.P. Seldin. Introduction to combinators and $\lambda$-calculus. London Mathematical Society Student Texts. Cambridge University Press, 1986.

[14] W.A. Howard. The formulae-as-types notion of construction. In J. P. Seldin and J. R. Hindley, editors, to H. B. Curry: Essays on Combinatory Logic, Lambda Calculus and Formalism, pages 479-490. Academic Press, 1980.

[15] S.C. Kleene. Introduction to metamathematics. North-Holland Publishing Company, 1954. Sixth Reprint (1971). 
[16] A.C. Leisenring. Mathematical Logic and Hilbert's $\epsilon$-Symbol. Gordon and Breach Science Publishers, New-York, 1969.

[17] C. R. Murthy. An evaluation semantics for classical proofs. In Proceedings of the sixth annual IEEE symposium on logic in computer science, pages 96-107, 1991.

[18] C. R. Murthy. A computational analysis of Girard's translation and LC. In Proceedings of the seventh annual IEEE symposium on logic in computer science, pages 90-101, 1992.

[19] M. Parigot. $\lambda \mu$-Calculus: an algorithmic interpretation of classical natural deduction. In A. Voronkov, editor, Proceedings of the International Conference on Logic Programming and Automated Reasoning, volume 624 of Lecture Notes in Artificial Intelligence, pages 190-201. Springer Verlag, 1992.

[20] D. Prawitz. Natural Deduction, A Proof-Theoretical Study. Almqvist \& Wiksell, Stockholm, 1965.

[21] S. Stenlund. Combinators $\lambda$-terms and proof theory. D. Reidel Publishing Company, 1972. 